\title{
Spontaneous assembly of subnanometre- ordered domains in the ligand shell of monolayer-protected nanoparticles
}

\author{
ALICIA M. JACKSON, JACOB W. MYERSON AND FRANCESCO STELLACCI* \\ Department of Materials Science and Engineering, MIT, 77 Massachusetts Avenue, Cambridge, Massachusetts 02139, USA \\ *e-mail: frstella@mit.edu
}

Published online: 18April 2004; doi:10.1038/nmat1116

The properties of materials can be created and improved either by confining their dimensions in the nanoscale or by controlling their nanostructure. We have combined these two concepts, and here we describe a new class of nanostructured nanosized materials that show ordered phase-separated domains at an unprecedented molecular length scale. Scanning tunnelling and transmission electron microscope images of monolayer-protected metal nanoparticles, with ligand shells composed of a mixture of molecules, show that the ligands phaseseparate into ordered domains as small as $5 \AA$. Importantly, the domain shape and dimensions can be controlled by varying the ligand composition or the metallic core size. We demonstrate that the formation of ordered domains depends on the curvature of the underlying substrate, and that novel properties result from this nanostructuring. For example, because the size of the domains is much smaller than the typical dimensions of a protein, these materials are extremely effective in avoiding non-specific adsorption of a variety of proteins.
$\mathrm{N}$ anoparticles $^{1-4}$, nanowires ${ }^{5,6}$ and nanotubes ${ }^{7}$ have recently attracted intensive research interest because of the uniqueness and ease in tailorability of their properties ${ }^{8}$. Similarly, nanostructured materials have shown improved properties (for example, the mechanical behaviour of nanostructured metals) ${ }^{9}$, as well as new ones (for example, the creation of a photonic bandgap in a block copolymer because of domain ordering $)^{10}$. The combination of nanosize and nanostructure can lead to a plethora of unique and complex materials. Indeed, nanowires composed of layers of different materials have shown selective and improved hole mobility ${ }^{11}$, and core-shell metal nanoparticles have tuneable surface plasmon resonance ${ }^{12}$. Here, we present a new class of materials: monolayer-protected metal nanoparticles ${ }^{1,3,4}(\mathrm{MPMN})$ with phase-separated ordered domains in their ligand shell. Because of the extremely small size of the domains $(\sim 5 \AA)$ these particles interact with the molecular environment in a novel way; for example, they prevent non-specific adsorption of proteins.

Self-assembled monolayers (SAMs) are monomolecular layers on surfaces ${ }^{13}$ that provide additional properties such as specific surface energies $^{14}$ and opto-electronic behaviour ${ }^{15}$. SAMs composed of a mixture of ligands can be easily produced in either one-step, by absorption from a solution of different molecules, or in two-steps, by placing a preformed monolayer into a solution of a different ligand ${ }^{13,16}$. Scanning tunnelling microscope (STM) images have shown that some mixed SAMs present phase-separated domains, but with no particular order ${ }^{16-20}$. It has been established that the phase separation is a thermodynamically driven process ${ }^{19}$.

MPMNs are supramolecular assemblies composed of a nanosized metallic core and an outer ligand shell, that is, a SAM on the metal surface $^{1}$. These particles show unique properties due to their core (for example, surface plasmon absorption $)^{21}$, to their ligands (solubility) ${ }^{1}$, or to both of their components (single electron transistor) ${ }^{22}$. They can be easily synthesised; in particular, the Schriffin method ${ }^{23}$ allows for straightforward control over the core size and ligand nature $\mathrm{r}^{1,23}$. An extension of this method-using one ${ }^{24}$ or two ${ }^{1}$ steps-leads to the synthesis of nanoparticles coated with a mixed $\mathrm{SAM}^{25,26}$. 


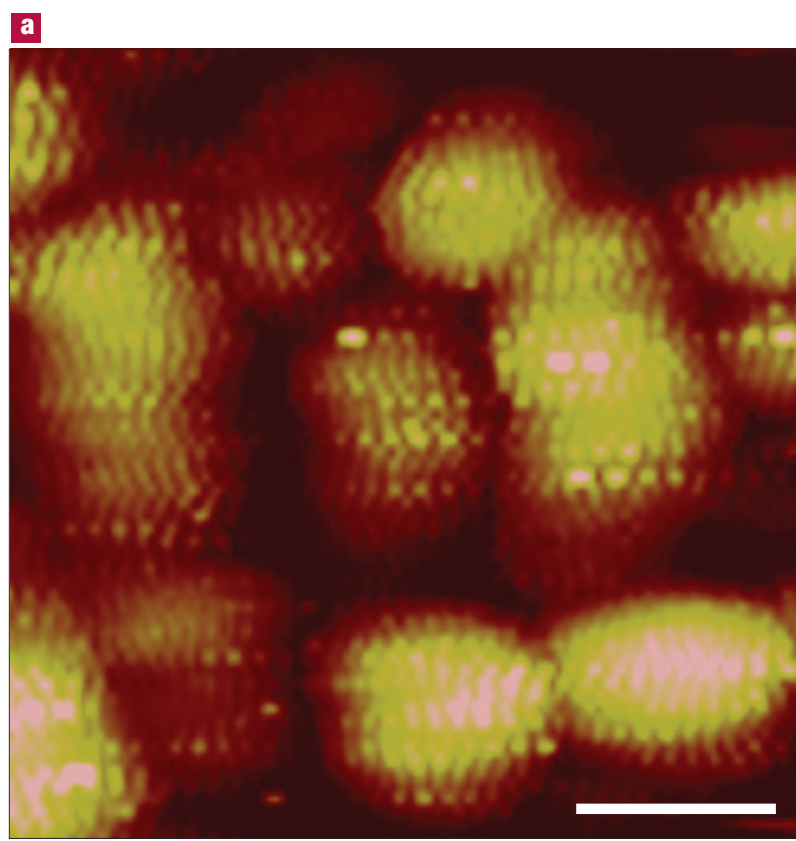

b

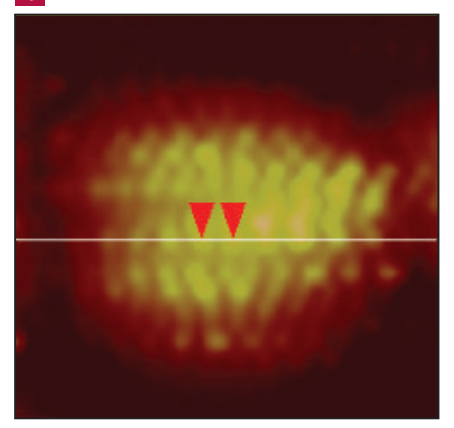

G

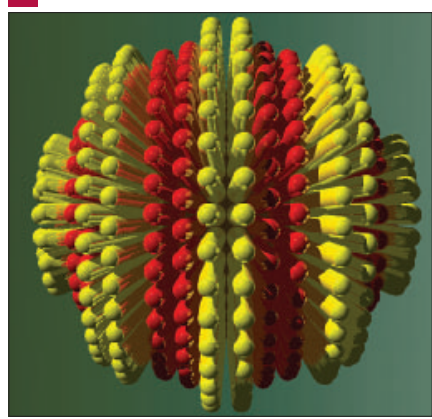

d

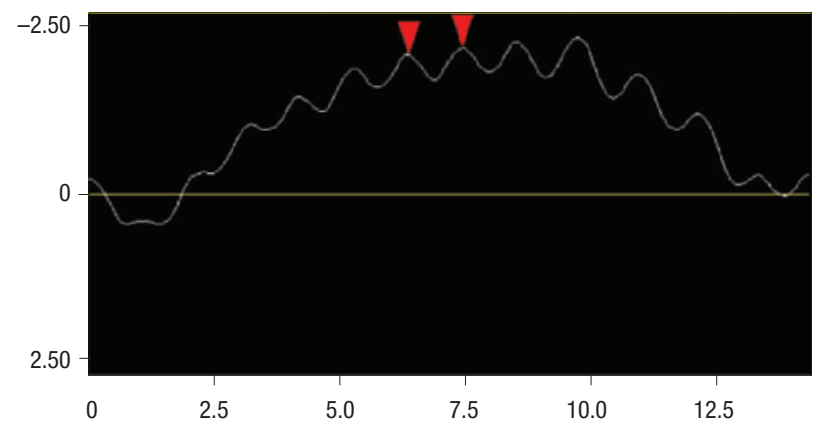

Figure 1 MPMNs with phase-separated ordered (rippled) domains on their ligand shell. a, STM image of 0T/MPA (2:1 molar ratio) gold nanoparticles showing phase-separated ripples on their ligand shell. Scale bar $10 \mathrm{~nm}$. b. STM image of one rippled nanoparticle. c, Schematic drawing of one single rippled nanoparticle in which the yellow lobes represent $0 T$ molecules and the red lobes represent MPA, shown to help the reader understand the morphology of the ligand shell. $\mathbf{d}$, Surface plot of the ligand shell contour showing the order that is present there (scales are in $\mathrm{nm}$ ). The surface plot was obtained from the nanoparticle shown in $\mathbf{b}$ at the white line. The distance between the two red arrows indicates the ripple spacing, using the convention chosen in this paper. Gold nanoparticles coated with 1-octanethiol $\left(\mathrm{CH}_{3}-\left(\mathrm{CH}_{2}\right)_{7}-\mathrm{SH}, \mathrm{OT}\right)$ and mercaptopropionic acid $\left(\mathrm{HOOC}-(\mathrm{CH})_{2}\right.$-SH, MPA) were synthesised in one step. The reaction procedure was similar to that described in the literature ${ }^{35}$, the only difference being that a mixture of 0T:MPA was used. STM samples were prepared by immersing a $1 \mathrm{~cm}^{2}$ gold substrate (either gold foil or Au(111) thermally evaporated on mica) in $20 \mathrm{ml}$ of a $5.6 \times 10^{-2} \mathrm{mM}$ toluene solution of 1,8-octane-dithiol containing $2 \mathrm{mg}$ nanoparticles for $24 \mathrm{~h}$. The dithiol was used to bind the nanoparticles to the substrate and to one another; this was found to be beneficial in imaging as the particles were immobilized and the STM tip could not be contaminated. Particular care was used in assuring that the observed nanostructures were not imaging artefacts.

First, the imaging was performed on particles synthesised on separate occasions; each particle batch was cast on different substrates and imaged with multiple tips on various days over a period of months. The resulting images all showed the same rippled nanoparticles with a variation in the peak-to-peak spacing of less then $10 \%$. Second, during each imaging session, images were collected at scan rates and scan sizes that covered a range of values from 3-6 Hz and 60-200 nm, respectively. Periodically, the scan direction was varied. Analysis of the collected images showed the peak-to-peak distance to vary less than $5 \%$. We attribute most of the variation to pixelate problems in the software. Finally, on numerous occasions the operator stopped the imaging of the mixed ligand nanoparticles and analysed homo-ligand nanoparticles always to find that in such cases either (i) no nanostructure or (ii) hexagonally packed head groups were visible, depending on the tip quality. As seen in a, it is evident that particle films present ripples aligned over relatively large length scales, forming long channels with hydrophilic bottoms and hydrophobic walls. It is known that the nanoparticle ligands interdigitate ${ }^{27,36}$. We believe that this phenomenon leads to the observed macroscopic alignment of the ripples across many nanoparticles. Indeed, it can be observed that there is no clear separation between the ligand shells of the nanoparticles. On heating above the de-interdigitation temperature and subsequent gentle cooling, we improved ripple alignment across the sample. Samples self-assembled on a gold foil from a 1,2-dichlorobenezene solution kept at a temperature $\left(150^{\circ} \mathrm{C}\right)$ above the de-interdigitation transition did not show consistent ripple alignment.

\section{NANOPARTICLE CHARACTERIZATION}

We have observed that subnanometre-ordered domains form spontaneously on the ligand shell of gold MPMNs coated with mixtures of ligands. This conclusion is based on the careful analysis of a large number of STM images of a variety of nanoparticles, and it is also based on the comparison of the parameters (shape and spacing) of the domains, and of the particle properties (solubility) across multiple targeted series of nanoparticles. As proven, for example, by the STM images shown in Fig. 1, gold particles synthesised in one step with a 2:1 OT/MPA molar composition (OT = octanethiol, $\mathrm{CH}_{3}-\left(\mathrm{CH}_{2}\right)_{7}-\mathrm{SH}$; and MPA = mercaptopropionic acid, HOOC- $\left(\mathrm{CH}_{2}\right)_{2}-\mathrm{SH}$,), present domains that align into parallel ripples that encircle and/or spiral around the nanoparticles. In contrast, STM images of homo-ligand nanoparticles lacked the rippled stripes and showed hexagonally packed head groups. The presence of ripples on the nanoparticles has also been confirmed using X-ray diffraction (XRD). Indeed, all of the XRD plots of the rippled nanoparticles described in this paper showed peaks at $2 \theta$ ranging from $2.5^{\circ}$ to $13^{\circ}$. Some of the peaks were temperature dependent, as is expected for peaks due to inter-particle packing arrangements ${ }^{27}$. However, one or two peaks were temperature independent, pointing to periodic arrangements, with $0.5-2.5 \mathrm{~nm}$ spacing, on single nanoparticles (see Supplementary Information, Fig. S1). Such temperature-independent peaks were never observed in homo-ligand nanoparticles. Additional confirmation of the presence of ordered phase-separated domains was provided by transmission electron microscopy (TEM) images. In fact, in these images (see 


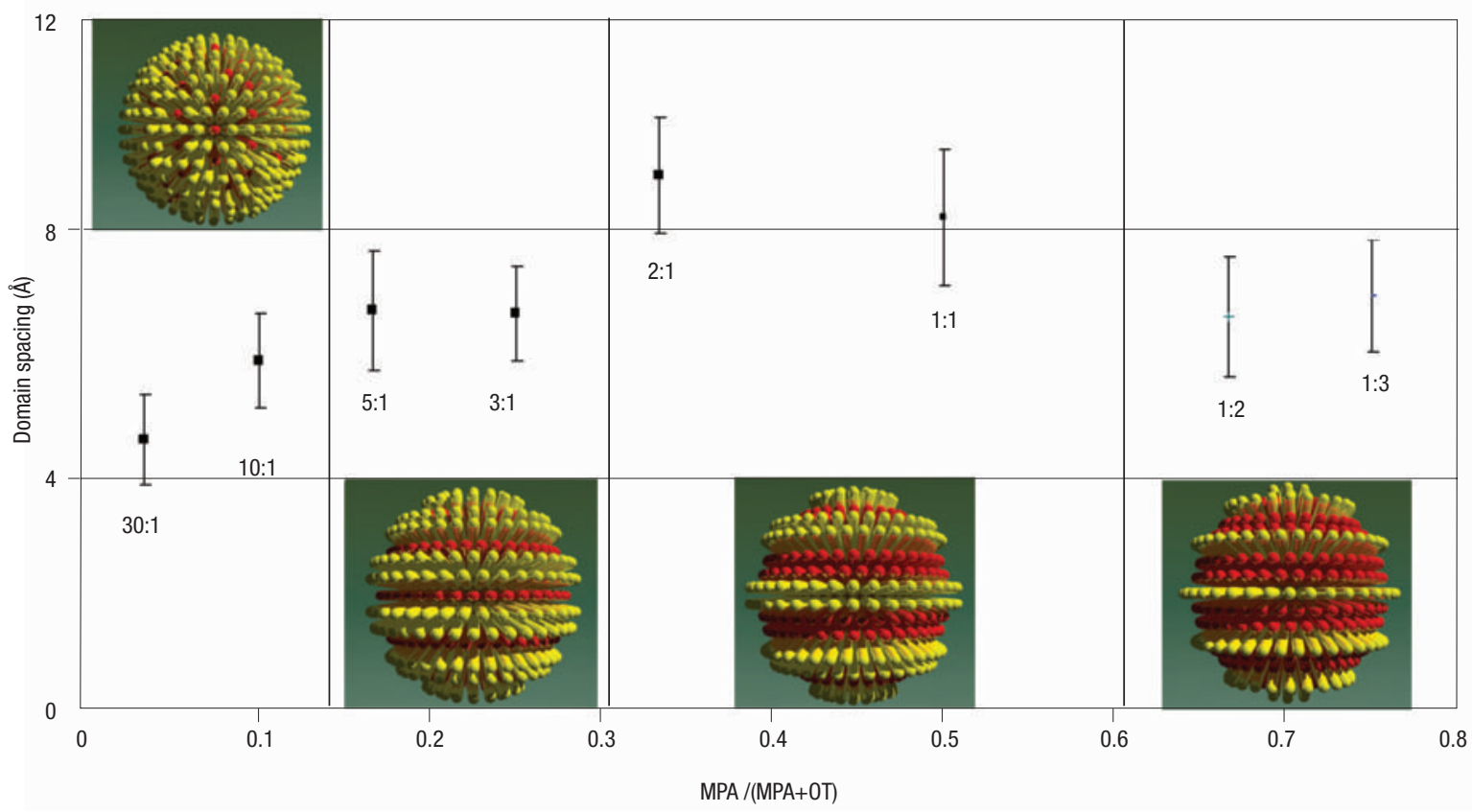

Figure 2 Plot of the domain spacing versus the MPA fraction used in the one-step synthesis of gold nanoparticles. All of the nanoparticles had an average diameter of $3.7 \mathrm{~nm}$. The fact that a variation in the spacing is observed points to a molecularly driven mechanism for the size control of the ripples and not to a metal-directed one. The schematic drawings of the particles are meant to help visualize a possible molecular origin of the discreteness and the trend in the spacing of the domains.

Supplementary Information, Fig. S2) we have found that there is an observable ring around the nanoparticles' metallic cores consisting of discrete dots spaced $\sim 0.5-0.6 \mathrm{~nm}$.

One of the most attractive properties of these nanoparticles is that the morphology of the ligand shell can be easily tailored. By simply varying the stoichiometry of the reagents during the one-step synthesis, it is possible to control and change the height difference, the spacing and the shape of the phase-separated domains, that is, the resulting exterior shape of the nanoparticle. We varied the height difference between the peaks and valleys of the ripples by synthesising nanoparticles with MPA and one type of n-alkane thiol $\left(\mathrm{CH}_{3}-\left(\mathrm{CH}_{2}\right)_{n}-\mathrm{SH}\right.$, where $\left.n=5,7,9,11\right)$ in a 1:2 molar ratio; all of the particles showed ripples and, as expected, the spacing remained constant. (Throughout this paper, the interdomain spacing is measured as the distance between one peak and the next one in an STM image, using image sections like Fig. 1c. It should be noted that this distance is a measure not of one single domain, but of the total width of one OT and one MPA domain.) We then varied the spacing between the ripples by first changing the metallic core size, while keeping the ligand (OT/MPA) ratio constant, and found that peak-to-peak distance decreases as the nanoparticle diameter increases. This is because the radius of curvature imposes the relative angle between one OT domain and the next, and this angle decreases with increasing core diameter. Even more strikingly, by varying the ligand ratio while keeping the core size constant, we found that we can change the peak-to-peak distance in quantized increments of $\sim 3 \AA$ (see Fig. 2). Changing the ligand ratio also proved to be a powerful tool for varying the global domain morphology, going from perfect ripples to defect-rich ripples to discrete domains. In the case of OT/MPA mixtures, we have observed that for extreme compositions in which one molecule is present in small amounts, discrete and ordered domains of the lesser component form in a percolated matrix of the more abundant one (see Fig. 3). This behaviour has some striking similarities to that of block copolymers ${ }^{28}$ (albeit at a much smaller length scale); indeed, one could think of the ripples as the lamellar phase. The dependence of the domain spacing on the nanoparticle core size and on the ligand stoichiometric ratio strongly confirms our theory of ordered phase-separation on the ligand shell of MPMNs.

The formation of phase-separated domains on nanoparticles is not confined to specific molecular mixtures or intermolecular forces. We synthesised a series of nanoparticles coated with alkane thiols and a longer carboxylic-acid-terminated thiolated molecule (mercapto undecanoic acid, HOOC- $\left.\left(\mathrm{CH}_{2}\right)_{11}-\mathrm{SH}, \mathrm{MUA}\right)$. All of the synthesised particles showed ordered domains. Nanoparticles that had a 2:1 molar ratio of dodecanethiol $\left(\mathrm{CH}_{3}-\left(\mathrm{CH}_{2}\right)_{11}-\mathrm{SH}\right)$ to MUA, two molecules that are approximately the same length, showed ripples, proving that the relative height difference is not the sole factor in determining ripple formation. Nanoparticles coated with a binary mixture of OT and 4-amino thiophenol $\left(\mathrm{H}_{2} \mathrm{~N}-\mathrm{C}_{6} \mathrm{H}_{4}-\mathrm{SH}\right)$ in a 1:1 molar ratio showed clear evidence of ripples with a $0.6-\mathrm{nm}$ spacing. This demonstrates that the phase-separated domains form also in the case of intermolecular forces such as $\pi-\pi$ interactions.

\section{NANOPARTICLE PROPERTIES}

We have started to investigate the consequences of nanostructuring on the properties of the nanoparticles. As shown in Fig. 4, domain morphology, not necessarily the ligand shell composition, determines the solubility of the particles. More interestingly, we have observed that these nanoparticles avoid non-specific adsorption of proteins. We believe that this happens because of the unique subnanometreordered repetition of hydrophobic and hydrophilic regions on the particles' ligand shell. The $5-\AA$ small domains on the outside of these particles are more than one order of magnitude smaller than the characteristic size of a protein globule; independently of the protein conformation, there will always be a series of attractive and repulsive 
a

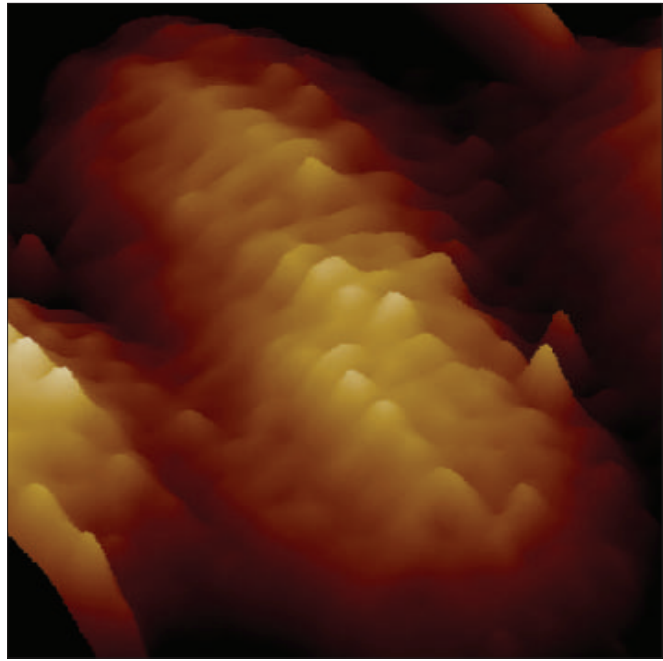

G

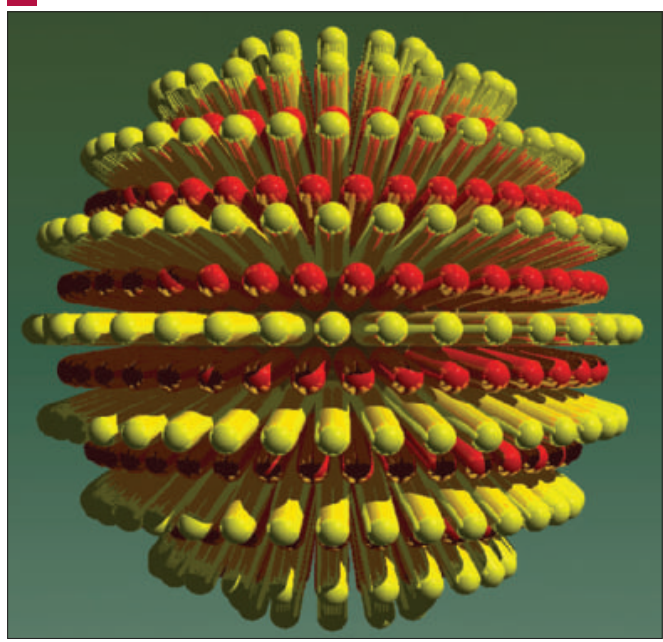

b

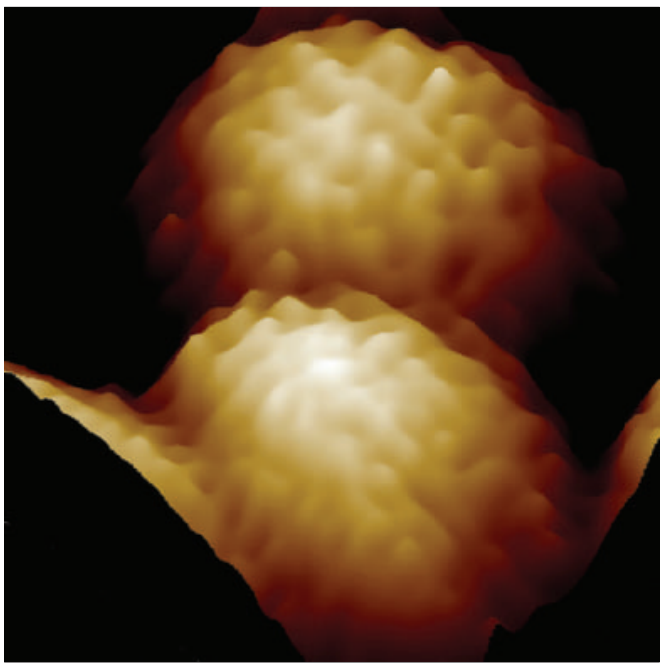

d

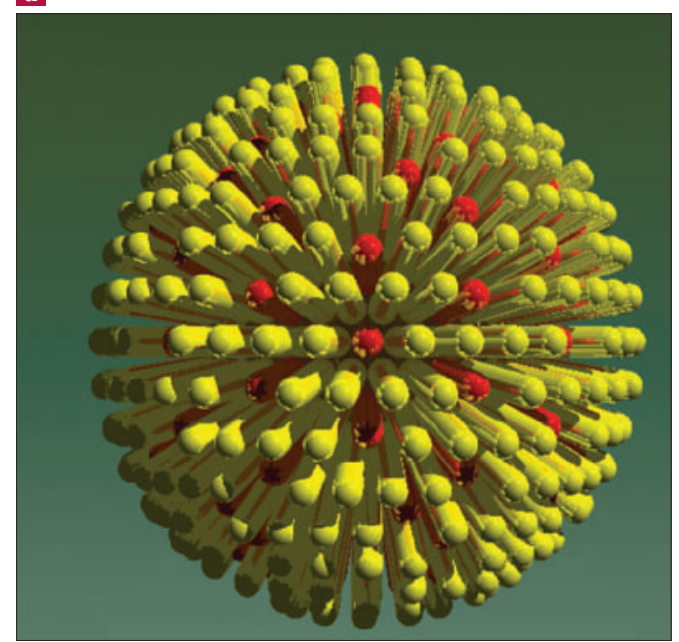

Figure 3 Three-dimensional rendering of STM height images of gold nanoparticles. a, Decanethiol/MPA (2:1 molar ratio) showing ripples and b, 0T/MPA (10:1 molar ratio) showing packed, phase-separated domains but not ripples. $\mathbf{c}, \mathbf{d}$, Schematic drawings of $\mathbf{a}$ and $\mathbf{b}$, respectively.

forces between the protein's outer shell and the particle's domains (see Fig. 5). Consequently, there will be almost no net attraction, and the protein adsorption from solution will not be thermodynamically favourable. To establish this relationship, we have chosen three proteins: (i) cytochrome $\mathrm{C}$, a medium-sized protein known to bind strongly to both OT and MPA homo- and mixed monolayers ${ }^{29}$, (ii) lysozyme, a small positively charged ( $\mathrm{pH} 7.4$ ) and rigid protein known not to unfold when adsorbing on hydrophilic monolayers ${ }^{30}$, and (iii) fibrinogen, a large protein present in blood plasma that adsorbs strongly to hydrophobic surfaces by unfolding ${ }^{31}$. Following known procedures ${ }^{29}$, (using STM, atomic force microscopy, and Fourier-transform infrared spectroscopy) we have confirmed that all of these proteins adsorb on MPA, OT and on mixed MPA/OT monolayers, and established that the same behaviour occurs on OT homo-ligand nanoparticle films, but the proteins do not adsorb on rippled or domained MPA/OT nanoparticle films, even after 24-h exposure to a concentrated solution.It should be pointed out that on rippled nanoparticle-coated surfaces we found no evidence for the presence of proteins with any of the characterization techniques used. We believe that this result is due to the unique size scale of the ordered hydrophobic and hydrophilic domains. These results are extremely promising, in fact, using three different characterization techniques we find that surfaces coated with nanostructured nanoparticles outperform surfaces that are known ${ }^{29-31}$ to have good protein-resistance properties with all of the three very different protein systems used.

\section{THE NANOSTRUCTURING OF SAMS}

Phase separation on this length scale had actually never been observed; this prompted us to investigate the ordering mechanism. To understand if the formation of the ripples depends on the nature of the metallic core, we synthesised silver nanoparticles with a 2:1 OT/MPA molar composition. The choice of silver was driven by the very similar chemistry between silver and gold. In fact, thiolated molecules are mobile on both metals, and the synthetic procedures to obtain silver and gold particles are very similar. Ripples formed with similar spacing to those on gold, suggesting that the ripple-formation mechanism is not 


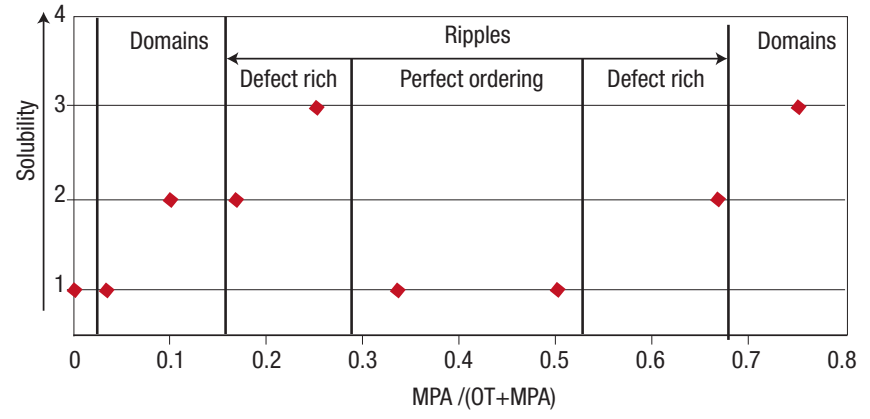

Figure 4 The solubility in ethanol of a series of OT/MPA gold nanoparticles as a function of MPA fraction ( $3.7 \mathrm{~nm}$ in diameter). It should be noted that the solubility does not monotonically increase with concentration of MPA as could have been expected from simple chemical arguments. In the region of perfect ripples, the longest ligand dominates the solubility behaviour. A more detailed description of this phenomenon can be derived from Table $\mathrm{S} 1$ in the Supplementary Information. All of the samples were prepared by combining $2 \mathrm{mg}$ nanoparticle with $20 \mathrm{ml}$ of ethanol and sonicating for 5 mins. The resulting mixture was then stirred for $5 \mathrm{~h}$, after which it was left undisturbed for 1 month. In the solubility scale, 4 = highly soluble, that is, no precipitation visually observed, $3=$ mostly soluble, that is, little precipitation observed over time with consequent slight decolouration of the solution; 2 = slightly soluble, that is, most of sample precipitated but a small coloration of the solution remains, 1 = totally insoluble.

solely determined by the core metal. Additionally, by comparing the TEM images of the metallic cores of MPMNs with the STM images of the ligand shells of the same particles, we conclude that the crystallographic nature of the metal is not a determining factor for ripple formation. In fact, $\sim 15 \%$ of the particles have cores that are twinned, whereas we have never observed 'twinned' domains on the particles' ligand shells. To prove that the ordering of the phase-domains is a result of a thermodynamic equilibrium, we prepared mixed-ligand nanoparticles in a two-step procedure starting from OT-coated nanoparticles and performing a place-exchange reaction with MPA. We observed ripples with the same spacing as those formed on nanoparticles synthesised in only one step. Because the same morphological end-point is reached from two different starting points, we conclude that the observed domains are an equilibrium state. This is additionally confirmed by the fact that the ripples on the nanoparticles are extremely stable; images of our samples taken over a period of more than eight months do not show any hint of change.

One of the possible causes of domain ordering is that the morphology of SAMs depends on the curvature of the substrate. We tested this by analysing SAMs on substrates of varying curvatures and by looking at nanoparticles of varying sizes. In the range of diameters in which nanoparticles are still soluble, we have not observed any loss of domain ordering. To produce a broader size and curvature range than that allowed by the synthetic and solubility constraints of MPMNs, we used surfaces covered with small gold hemispheres of varying diameters. Mixed SAMs were formed on gold thermally evaporated on silicon, which presents a high density of 20-nm-diameter hemispheres of, on average, $5 \mathrm{~nm}$ in height. Because of the low curvature of the substrate, there was no domain ordering. To better approximate the curvature present on nanoparticle surfaces, nanoparticle submonolayers were prepared on gold on mica substrates and then processed so as to completely remove their ligands, resulting in gold hemispheres $\sim 4 \mathrm{~nm}$ in diameter and height. When mixed monolayers were assembled on these surfaces, ordered ripples spontaneously formed only on the curved part of the substrate, identifying curvature as the cause for ripple formation (see Fig. 6). Further studies need to be
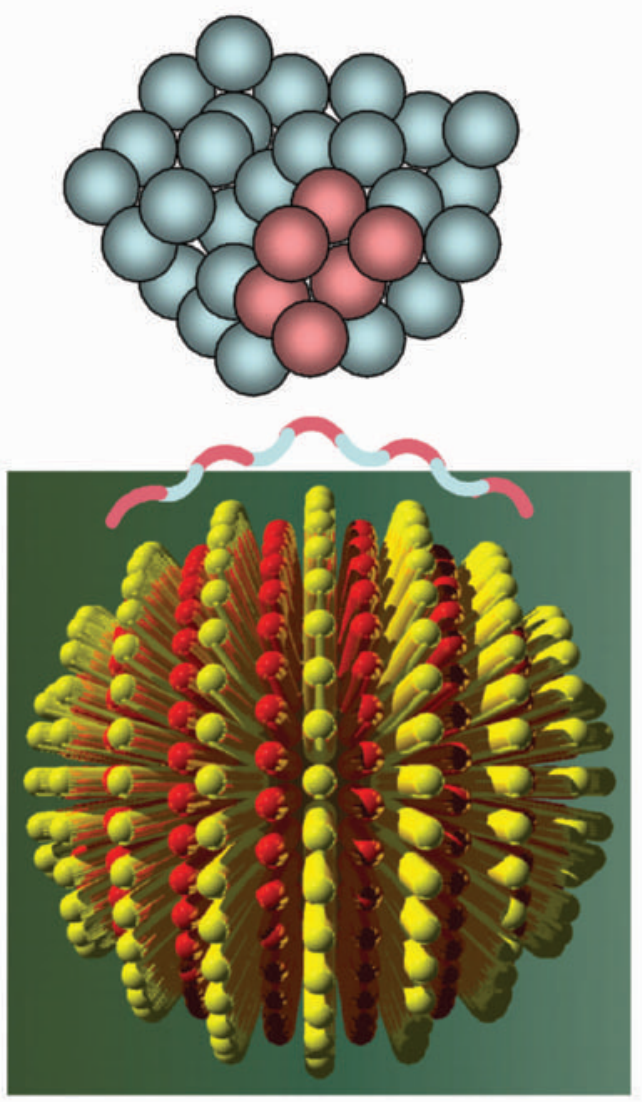

Figure 5 Schematic drawing of a generic protein (top) and a rippled nanoparticle (bottom). The pink and blue contour line on top of the nanoparticle shows the hydrophobic and the hydrophilic regions of the particle, respectively. The same colour scheme is used to show the outside shell of the chosen protein. It is evident (as the drawing is approximately to scale) how, despite the enormous conformational freedom that the proteins have, there will always be regions of attraction and regions of repulsion when interacting with nanostructured nanoparticles.

done in order to understand if curvature determines this phenomenon because of geometrical conditions ${ }^{32}$, anisotropic surface stress $^{33}$, or mechanisms that are yet to be understood.

Ripples on rough surfaces and on nanoparticles synthesised in two steps were full of defects, whereas ripples formed on nanoparticles synthesised in one step were almost defect-free. It has been established ${ }^{34}$ that (AuSR) ${ }_{n}$, where $\mathrm{R}$ is the alkyl chain, (present in the two-step syntheses and absent in the one-step) acts as a catalyst, able to increase ligand place-exchange reaction rates. Consequently, we formed mixed monolayers on gold on mica and rough gold on silicon surfaces in the presence of (AuSR) ${ }_{n}$. In the case of flat gold on mica, phase-separated domains with no order were observed, similar to those that formed in the absence of (AuSR) ${ }_{n}$. However, mixed monolayers on gold on silicon showed ripples only when prepared in the presence of (AuSR) $n$, indicating that kinetic rates have an important role in the ordering of the nanoscale domains.

In conclusion, we show for the first time that gold and silver nanoparticles coated with mixtures of ligands present nanostructuring in their ligand shells due to phase separation driven by surface curvature. The morphology of the nano-structures within the ligand shell, and consequently the properties, can be controlled by varying the 
a
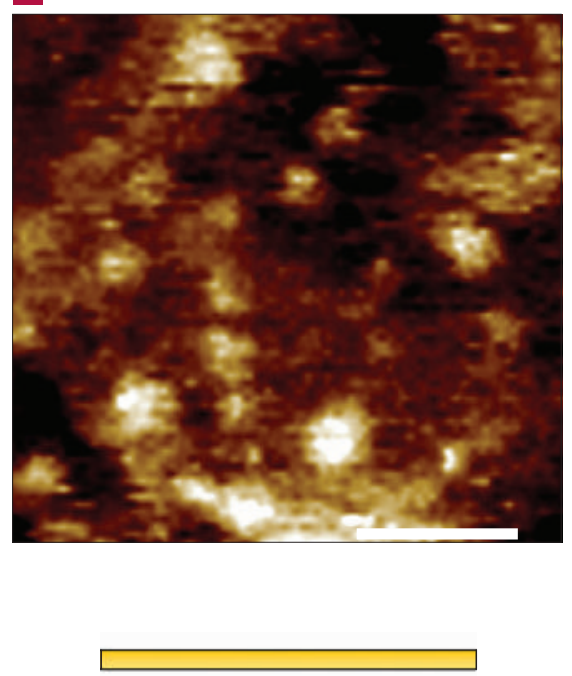

G
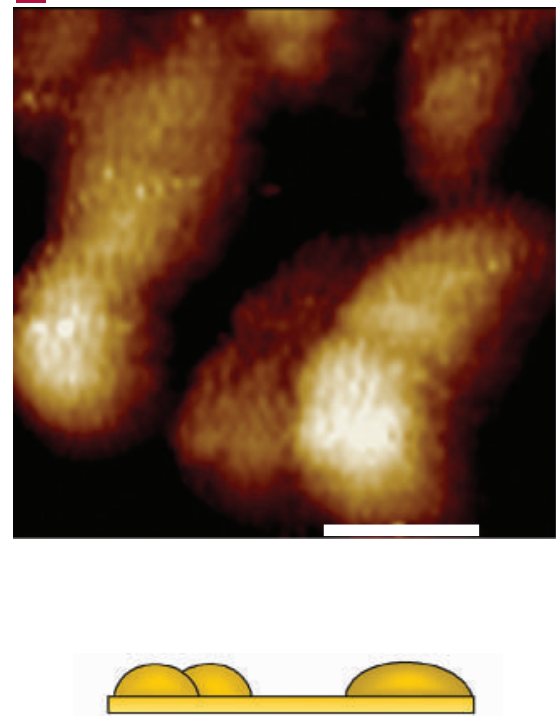

b
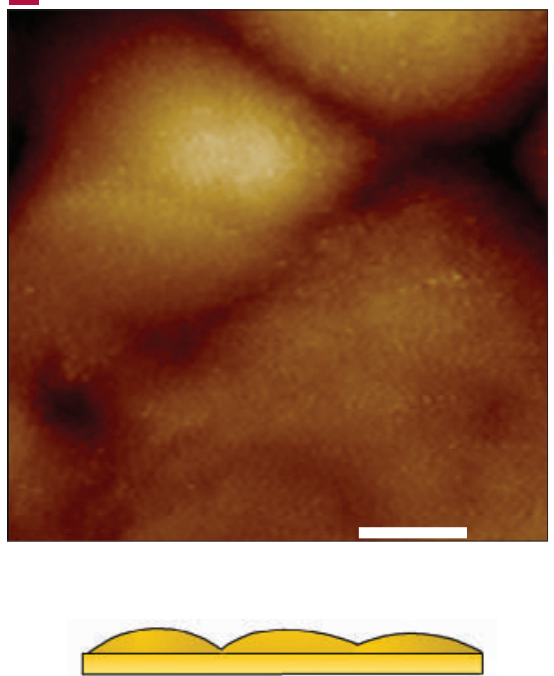

d
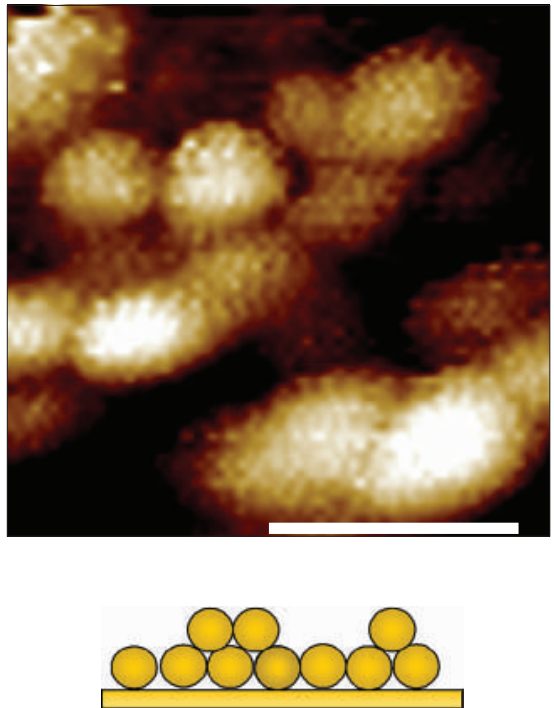

Figure 6 STM images of mixed OT/MPA monolayers formed on surfaces of varying curvature. Drawings of the gold surface curvature are shown underneath the STM height images of mixed monolayers formed on $\mathbf{a}$, a flat $A u(111)$ on mica substrate; $\mathbf{b}$, a gold film deposited on a silicon wafer showing hemispheres of $\sim 20 \mathrm{~nm}$ diameter; $\mathbf{c}$, a gold film containing gold crystals of $\sim 10 \mathrm{~nm}$ diameter; $\mathbf{d}$, a gold film with 5 -nm gold crystals on it. Ordered, rippled domains are observed only in $\mathbf{c}$ and $\mathbf{d}$, whereas in $\mathbf{a}$ and $\mathbf{b}$, randomly dispersed domains are observed. All scale bars are $10 \mathrm{~nm}$. All the monolayers were obtained from a $1 \mathrm{mM}$ ethanol solution of OT/MPA (1:4 mol/mol). Substrates c and $\mathbf{d}$ were obtained by first forming a homoligand (OT) nanoparticle submonolayer on a clean gold on mica substrate and then by removing the ligands from the nanoparticles. Specifically, $\mathbf{C}$ was heated under vacuum at $170^{\circ} \mathrm{C}$ for $1 \mathrm{~h}$ and $\mathbf{d}$ was irradiated with ultraviolet light for $8 \mathrm{~h}$. Both samples were then cleaned and imaged with STM, showing absence of ligands. Gold islands comparable in size and shape to the metallic cores of the nanoparticles, albeit slightly larger, and in the case of $\mathbf{c}$, flatter, were observed. It should be noted that on the flat regions of the sample $\mathbf{d}$, the ligands phase-separated in a random way, resembling the behaviour shown in a. The fact that no noticeable difference was observed between surfaces generated in different methods seems to rule out a strong effect of the specific crystallographic nature of the metal surface.

molar ratio and the nature of the ligands. We believe that these particles will have significant applications in nanotechnology and biology.

\section{METHODS}

All of the chemicals and gold foils were purchased from Sigma-Aldrich and used as received. Au(111) thermally evaporated on mica was purchased from Molecular Imaging. Mechanically cut Pt-Ir STM tips were obtained from Digital Imaging. STM images were obtained using a Digital Instrument Multimode Nanoscope IIIA with a bias voltage $1,000 \mathrm{mV}$ and tunnelling current of 500 to $700 \mathrm{pA}$. TEM images were made on a JEOL 2010. X-Ray data were obtained from a Rigaku $185 \mathrm{~mm}$ diffractometer.

\section{NANOPARTICLE SYNTHESIS}

Nanoparticles were synthesised using a slightly modified version of the Schriffin method. The general procedure was to dissolve $354 \mathrm{mg}(0.9 \mathrm{mmol})$ of $\mathrm{HAuCl}_{4} 3 \mathrm{H}_{2} \mathrm{O}$ in $50 \mathrm{ml}$ of water and $2.187 \mathrm{~g}(4 \mathrm{mmol})$ of $\mathrm{BrN}\left(\left(\mathrm{CH}_{2}\right)_{7} \mathrm{CH}_{3}\right)_{4}$ in $80 \mathrm{ml}$ of toluene. The two phases were mixed and left stirring for $30 \mathrm{~min}$. Mixtures of the targeted ligands in the molar amounts specified in Table S1 in the Supplementary Information were injected in the solution once the colour due to the gold salt had transferred completely to the organic phase. The solution was allowed to react for ten minutes and acquired a typical white colour. A $10 \mathrm{mM}$ solution $(30 \mathrm{ml})$ of $\mathrm{NaBH}_{4}$ was then added dropwise over $1 \mathrm{~h}$. After the addition, the solution was left stirring for $2 \mathrm{~h}$. The phases were separated and the organic phase was collected, reduced to $10 \mathrm{ml}$, diluted with $100 \mathrm{ml}$ of absolute ethanol, and placed in a refrigerator overnight. The precipitate was collected by vacuum filtration using quantitative paper filters and extensively washed with water, acetone 
and ethanol. Typically, the collected black powder would weigh $\sim 100 \mathrm{mg}$. Nanoparticles soluble in ethanol were collected by vacuum evaporation of the ethanol solution and extensive rinsing with water, acetone and toluene.

Silver nanoparticles were synthesised by dissolving $152.9 \mathrm{mg}(0.9 \mathrm{mmol})$ of $\mathrm{AgNO}_{3}$ in $100 \mathrm{ml}$ of ethanol followed by the addition of mixtures of the targeted ligands in the molar amounts specified in Table S1 in the Supplementary Information. The solution was kept at $0{ }^{\circ} \mathrm{C}$. After $10 \mathrm{~min}$ a saturated solution (100 ml) of $\mathrm{NaBH}_{4}$ was slowly added dropwise. After completion, the solution was stirred for $2 \mathrm{~h}$, brought to room temperature, and placed in a refrigerator overnight. The purification was the same as that described for gold nanoparticles.

A detailed description of the synthesis of each single nanoparticle will be published elsewhere.

\section{MONOLAYER FORMATION AND SURFACE PREPARATION}

Self-assembled monolayers were prepared on $\mathrm{Au}(111)$ by immersing the substrate in a $1 \mathrm{mM}$ thio solution for more than $24 \mathrm{~h}$, followed by rinsing with absolute ethanol and drying in air. Mixed monolayers of OT and MPA were prepared using $x_{\mathrm{MPA}}=0.80$, where $x_{\mathrm{MPA}}=c_{\mathrm{MPA}} /\left(c_{\mathrm{MPA}}+c_{\mathrm{OT}}\right)$ and $c_{\mathrm{i}}$ denotes the molar concentration of $\mathrm{i} ; c_{\mathrm{MPA}}+c_{\mathrm{OT}}=1 \mathrm{mM}$. Cytochrome $\mathrm{C}$ was adsorbed on the SAMs by immersing the monolayers for $24 \mathrm{~h}$ in a solution of $137 \mathrm{mmol} \mathrm{NaCl}, 2.7 \mathrm{mmol} \mathrm{KCL}$, and $10 \mathrm{mmol}$ phosphate buffer (200 $\mathrm{ml} \mathrm{H}_{2} \mathrm{O}$, pH 7.4) containing $197.6 \mathrm{mg}(0.016 \mathrm{mmol})$ Cytochrome C. Lysozyme adsorption was performed by incubating the substrates in $0.1 \mathrm{mg}$ lysozyme/ $1 \mathrm{ml} \mathrm{PBS}$ for $24 \mathrm{~h}$. Fibrinogen adsorption was performed by incubating the substrates in a $0.5 \mathrm{mg}$ fibrinogen/ $1 \mathrm{ml}$ PBS for $24 \mathrm{~h}$. All substrates were subsequently thoroughly rinsed with PBS and purified water and dried under air.

SAMs formed in the presence of (AuSR) ${ }_{n}$ were prepared by immersing gold on mica and gold on silicon substrates into a $1 \mathrm{mM}$ ethanol solution of OT/MPA (2:1 mol/mol) for $2 \mathrm{~h} . \mathrm{HAuCl}_{4}(1 \mathrm{mM})$ was then added to the solution and stirred overnight. The substrates were then rinsed extensively with toluene and ethanol, followed by drying with nitrogen.

The heat treatment of nanoparticle monolayers was accomplished by forming a nanoparticle film on a gold substrate as previously described, rinsing with toluene, and heating to $120^{\circ} \mathrm{C}$ for $1 \mathrm{~h}$. The substrates were then cooled to room temperature, followed by successive rinsing with toluene, dichloromethane, ethanol, acetone and acetonitrile.

To create a surface of high curvature, one nanoparticle monolayer, prepared as described above, was placed in a round-bottom flask under vacuum $\left(10^{-3}\right.$ torr $)$ and then heated at $170^{\circ} \mathrm{C}$ for $1 \mathrm{~h}$. The flask was brought back to atmospheric pressure and allowed to cool to room temperature. The sample was then cleaned with acetone and imaged with STM. Another nanoparticle submonolayer was irradiated with ultraviolet light ( $21.7 \mathrm{~mW} \mathrm{~cm}^{-2}$, B-100 AP, Fisher Scientific) for $8 \mathrm{~h}$; with subsequent rinsing with acetone, and then imaged with STM.

Mixed ligand OT/MPA nanoparticles synthesised by the two-step synthesis were first synthesised as OT homoligand nanoparticles by the above one-step procedure. $50 \mathrm{mg}$ of the resulting nanoparticle were then dissolved in $45 \mathrm{ml}$ toluene and $5 \mathrm{ml}$ of MPA. The solution was stirred for $24 \mathrm{~h}$, followed by centrifugation to remove unsubstituted ligands.

\section{Received 9 December 2003; accepted 15 March 2004; published 18 April 2004.}

\section{References}

1. Templeton, A. C., Wuelfing, M. P. \& Murray, R. W. Monolayer protected cluster molecules. Acc. Chem Res. 33, 27-36 (2000)

2. Alivisatos, A. P. Semiconductor clusters, nanocrystals, and quantum dots. Science 271, 933-937 (1996).

3. Daniel, M. C. \& Astruc, D. Gold nanoparticles: Assembly, supramolecular chemistry, quantum-sizerelated properties, and applications toward biology, catalysis, and nanotechnology. Chem. Rev. 104 293-346 (2004).

4. Thomas, K. G. \& Kamat, P. V. Chromophore-functionalized gold nanoparticles. Acc. Chem. Res. 36, 888-898 (2003).

5. Xia, Y. N. et al. One-dimensional nanostructures: Synthesis, characterization, and applications. $A d v$ Mater. 15, 353-389 (2003)

6. Hu, J. T., Odom, T. W. \& Lieber, C. M. Chemistry and physics in one dimension: Synthesis and properties of nanowires and nanotubes. Acc. Chem. Res. 32, 435-445 (1999).

7. Dai, H. J. Carbon nanotubes: Synthesis, integration, and properties. Acc. Chem. Res. 35, 1035-1044 (2002).

8. Empedocles, S. A., Neuhauser, R., Shimizu, K. \& Bawendi, M. G. Photoluminescence from single semiconductor nanostructures. Adv. Mater. 11, 1243-1256 (1999)

9. Schiotz, J., Di Tolla, F. D. \& Jacobsen, K. W. Softening of nanocrystalline metals at very small grain sizes. Nature 391, 561-563 (1998).

10. Bockstaller, M., Kolb, R. \& Thomas, E. L. Metallodielectric photonic crystals based on diblock copolymers. Adv. Mater. 13, 1783-1786 (2001).

11. Lauhon, L. J., Gudiksen, M. S., Wang, C. L. \& Lieber, C. M. Epitaxial core-shell and core-multishell nanowire heterostructures. Nature 420, 57-61 (2002).
12. Oldenburg, S. J., Hale, G. D., Jackson, J. B. \& Halas, N. J. Light scattering from dipole and quadrupole nanoshell antennas. Appl. Phys. Lett. 75, 1063-1065 (1999).

13. Ulman, A. Formation and structure of self-assembled monolayers. Chem. Rev. 96, 1533-1554 (1996)

14. Bain, C. D. \& Whitesides, G. M. Modeling organic-surfaces with self-assembled monolayers. Angew. Chem. Intl Edn 28, 506-512 (1989).

15. Yitzchaik, S. \& Marks, T. J. Chromophoric self-assembled superlattices. Acc. Chem. Res. 29, 197-202 (1996).

16. Stranick, S. J. et al. Nanometer-scale phase separation in mixed composition self-assembled monolayers. Nanotechnology 7, 438-442 (1996)

17. Delamarche, E., Michel, B., Biebuyck, H. A. \& Gerber, C. Golden interfaces: The surface of selfassembled monolayers. Adv. Mater. 8, 719-724 (1996).

18. Folkers, J. P., Laibinis, P. E. \& Whitesides, G. M. Self-Assembled monolayers of alkanethiols on gold comparisons of monolayers containing mixtures of short-chain and long-chain constituents with $\mathrm{CH}_{3}$ and $\mathrm{CH}_{2} \mathrm{OH}$ terminal groups. Langmuir 8, 1330-1341 (1992).

19. Smith, R. K. et al. Phase separation within a binary self-assembled monolayer on $\mathrm{Au}\{111\}$ driven by an amide-containing alkanethiol. J. Phys. Chem. B 105, 1119-1122 (2001).

20. Imabayashi, S., Gon, N., Sasaki, T., Hobara, D. \& Kakiuchi, T. Effect of nanometer-scale phase separation on wetting of binary self-assembled thiol monolayers on $\mathrm{Au}(111)$. Langmuir 14, 2348-2351 (1998)

21. Link, S. \& El-Sayed, M. A. Spectral properties and relaxation dynamics of surface plasmon electronic oscillations in gold and silver nanodots and nanorods. J. Phys. Chem. B 103, 8410-8426 (1999).

22. Andres, R. P. et al. "Coulomb staircase" at room temperature in a self-assembled molecular nanostructure. Science 272, 1323-1325 (1996).

23. Brust, M., Walker, M., Bethell, D., Schiffrin, D. J. \& Whyman, R. Synthesis of thiol-derivatized gold nanoparticles in a 2-phase liquid-liquid system. J. Chem. Soc. Chem. Commun. 801-802 (1994).

24. Stellacci, F. et al. Ultrabright supramolecular beacons based on self-assembly of two-photon chromophores on metal nanoparticles. J. Am. Chem. Soc. 125, 328-328 (2003).

25. Ingram, R. S., Hostetler, M. J. \& Murray, R. W. Poly-hetero-omega-functionalized alkanethiolatestabilized gold cluster compounds. J. Am. Chem. Soc. 119, 9175-9178 (1997)

26. Stellacci, F. et al. Laser and electron-beam induced growth of nanoparticles for 2D and 3D metal patterning. Adv. Mater. 14, 194-198 (2002).

27. Sandhyarani, N., Pradeep, T., Chakrabarti, J., Yousuf, M. \& Sahu, H. K. Distinct liquid phase in metal cluster superlattice solids. Phys. Rev. B 62, R739-R742 (2000).

28. Fasolka, M. J. \& Mayes, A. M. Block copolymer thin films: Physics and applications. Annu. Rev. Mater. Res. 31, 323-355 (2001).

29. Hobara, D., Imabayashi, S. \& Kakiuchi, T. Preferential adsorption of horse heart cytochrome C on nanometer-scale domains of a phase-separated binary self-assembled monolayer of 3mercaptopropionic acid and 1-hexadecanethiol on $\mathrm{Au}(111)$. Nano Lett. 2, 1021-1025 (2002).

30. Satulovsky, J., Carignano, M. A. \& Szleifer, I. Kinetic and thermodynamic control of protein adsorption. Proc. Natl Acad. Sci. USA 97, 9037-9041 (2000).

31. Kidoaki, S. \& Matsuda, T. Adhesion forces of the blood plasma proteins on self-assembled monolayer surfaces of alkanethiolates with different functional groups measured by an atomic force microscope. Langmuir 15, 7639-7646 (1999)

32. Nelson, D. R. Toward a tetravalent chemistry of colloids. Nano Lett. 2, 1125-1129 (2002).

33. Lu, W. \& Suo, Z. Symmetry breaking in self-assembled monolayers on solid surfaces. II. Anisotropic substrate elasticity. Phys. Rev. B 65 (2002).

34. Song, Y., Huang, T. \& Murray, R. W. Heterophase Ligand Exchange and Metal Transfer between Monolayer Protected Clusters. J. Am. Chem. Soc. 125, 11694-11701 (2003).

35. Terrill, R. H. et al. Monolayers in three dimensions: NMR, SAXS, thermal, and electron hopping studies of alkanethiol stabilized gold clusters. J. Am. Chem. Soc. 117, 12537-12548 (1995) 36. Badia, A. et al. Self-assembled monolayers on gold nanoparticles. Chem. Europ. J. 2, 359-363 (1996).

\section{Acknowledgements}

This work was supported in part by the Material Research Science and Education Center Program of the National Science Foundation under award number DMR 02-13282, and made use of its shared facilities. It was also supported by NIRT DMR-0303973 of the National Science Foundation. J.W.M. acknowledges support by the P. E. Gray fund for undergraduate research. The authors are extremely grateful to Blaise Gassend for his contribution to the synthesis of nanoparticles. Mike Frongillo is acknowledged for hi invaluable assistance with the TEM images. Dave Voci, Digital Instruments, is acknowledged for his continuing support.

Correspondence and requests for materials should be addressed to F.S.

Supplementary Information accompanies the paper on www.nature.com/naturematerials

\section{Competing financial interests}

The authors declare that they have no competing financial interests. 